DOI: $10.19195 / 2084-5065.49 .2$

\title{
Forensic examination of anonymous handwritten documents in order to obtain data on the identity of their author and performer
}

\author{
DMITRY V. BAKHTEEV \\ ORCID: 0000-0002-0869-601X \\ Criminalistics Department \\ Ural State Law University \\ Yekaterinburg, Russia \\ ALEXEY V. ANTROPOV \\ ORCID: 0000-0002-4702-753X \\ Criminalistics Department \\ Ural State Law University \\ Yekaterinburg, Russia
}

The information field of a handwritten document is quite large and diverse. Its contents include various elements, the study of which is the task of various areas of forensic knowledge. The letter is not only a means of communication, but also a means of transmission of criminally relevant information. For a full study of the document in order to clarify the most complete and reliable information about the persons involved in its creation, it is necessary to use an integrated approach. A handwritten document, as a carrier of information about a person, is a set of two elements: handwriting and writing. 
Handwriting is a set of writing and motor skills, characterizing the graphic technique of writing. On the one hand, the handwriting is a carrier of information about the person which can solve the identification problem of determination of a specific performer. In addition, the handwriting displays both the psychophysiological qualities of the writing person and the conditions in which the writing process took place. That is, the handwriting contains uniquely coded information about the person, the circumstances of writing and the conditions of the manuscript. The study of handwriting is engaged in handwriting, and the study of specific samples of handwriting is carried out during handwriting examinations.

Written speech represents a set of linguistic means used to express the thoughts of the author of the document. The study of written speech is engaged in diagnostic and identification linguistic studies.

An additional element can also be a document as a material object a sheet of paper and ink on it. In this case, in addition to forensic methods, the achievements of physics and chemistry can be used for its study (for example, the use of gas chromatography to establish the age of the document).

In this paper, we consider some methods of handwriting and linguistic studies of anonymous handwritten documents in order to establish the general and specific features of its performer and/or author.

The possibility of establishing the author and performer of the document is based on the uniqueness and stability of the written-motor and speech skills. The process of formation of the letter is influenced by the conditions of human learning, the environment, labor and social activities, psychophysiological and cognitive abilities.

The stability of the manifestation of writing skills largely depends on the depth of fixation of the motor skill, its automation in the higher nervous system, that is, the stronger the depth of fixation of the skill, the more difficult the change in the written-motor functions of a certain person.

The automatization of the writing is pronounced only while it is performed in the usual (stereotyped) conditions. An abrupt change of conditions of the letter (its target setting, physical and psychological complications, intentional distortion of the written speech and handwriting) leads to the termination or weakening of automatization of the writing as it is carried out under the strengthened control of consciousness. But in view 
of the short duration of these factors, the established manner of writing does not disappear and is only temporarily "blunted". When the usual conditions are restored - automation skills "wake up" again. This phenomenon is observed even when intentional attempts to create changes in handwriting and written language are made. At the same time, personal skills, fixed by the developed stereotype, remain, and have an impact on the process of creating the document.

The initial study of written documents can be carried out in the production of investigative actions, which in the Russian Federation is carried out in the presence of witnesses or fixation using technical means and is accompanied by the preparation of a procedural document - a protocol.

The results of such investigations or judicial proceedings acquire evidentiary value. This form of work with the document is called procedural, but at the same time, there is a non-procedural form of personal study of documents. It is called a preliminary study, the result of which may be the making of organizational or procedural decisions by the investigator: the organization of a forensic examination, the use of the document in proving the circumstances of the case and the production of interrogation or other investigative action.

Despite the variety of forms and types of documents and their content, in the course of their study typical tasks are solved, the main of which are:

1. Correct procedural registration of the fact of detection and withdrawal of the document as material evidence;

2. Individualization of the document by its description, photo and video, digital scanning;

3. Definition (selection, evaluation) of the main properties, qualities of the document: its type (private, official), purpose, origin, belonging to someone, the presence of requisites, compliance with the form and content, appearance and condition of the document;

4. Definition of the topographic features of letters (the locations of the text as a whole or its individual parts).

This class of signs allows us to judge the accuracy of the person. Often these signs are formed as a result of professional activity, for example, law enforcement officers in the Russian Federation automatically leave the left margin of $2 \mathrm{~cm}$ on paper when writing. 
These topographic features include:

- fields (left and right, size, rectangular and trapezoidal are most often vividly peculiar to people whose job functions are related to office work);

- the presence and size of indentations (used for structuring the document, characteristic of people who are professionally engaged in journalism, literature, scientific publications and other creative activities related to writing);

— the size of the line and word spacing;

- line direction: horizontal, ascending, descending, rectilinear, convex, concave, curved, stepped;

- the location of punctuation and hyphenation, the position titles, addresses and page numbers.

The topographical features of letters are stable and little controlled by the performer.

5. The specification of specific features of manufacture, storage, methods of making changes in the requisites and the contents of the document, the presence of encrypted records, etc. Such special features of document include:

- separate parts of the text (indicates the habit of the author or performer of the document to focus on the main ideas, public speaking experience);

- correction (allows judgment of the accuracy of the person, his literacy and conditions of creating the document [haste, a strong excitement, fatigue]);

- the presence or absence of signs of transfer (literacy, individual habits of paperwork);

- the originality of the date of writing (influenced by area of residence, native language). E.g., in Russian it is customary to write the date as Day-Month-Year, while in English it is Month-Day-Year).

6. Determination of general features of handwriting, such as:

- the proficiency of handwriting (the ability to use cursive writing, the pace of writing, coordination of movements);

- the complexity of the handwriting (simple and complex, including stylized);

- the general form of the direction of movement in the handwriting (rectilinear and arc, angular, rounded); 
- direction of separate movements: left and right;

- inclination of handwriting (direct, right-inclined, left-inclined, oblique);

- handwriting size: large - more than $5 \mathrm{~mm}$, medium $-2-5 \mathrm{~mm}$, small — less than $2 \mathrm{~mm}$;

- acceleration of handwriting - compressed, medium, sweeping;

- coherence of handwriting (continuous - 6 or more letters, average - 3-4 letters, interval - 1-2 letters);

- pressure (strong, medium and weak).

Forensic examination of anonymous texts to obtaining data on the identity of the author and the performer should begin with a thorough visual inspection of the document to find traces of hands. If the document is in the envelope, the investigator must set by the postmark where and when it was sent.

When examining the materials of the document and the handwritten text, it is also necessary to pay attention to the following:

- whether a rare type of paper or a writing instrument was used;

- the configuration of the separating line of the paper sheet (cut or tear);

- if the document has any impressed or scrapped traces of other records;

— the placement of independent fragments of documents;

- the ways to emphasize words, highlight individual words, add inserts, correct errors in words;

- the ways to transfer words, page numbering.

All this allows us to obtain orienting data about the habits of the text performer. After preliminary examination of the document, an analysis of its content is performed, namely:

1. The theme of the manuscript is analyzed, as is the purpose of its implementation;

2. It is determined by what factual data had the author of the document (for example, what certain events are known to him, with which persons he is familiar).

Then, the following is determined:

1. Is the handwritten text sufficient for lexical and stylistic analysis (it usually requires at least 2 pages of handwritten text)? 
2. Are there any intentional changes in the text and grammar to imitate the speech of illiterate persons of a low cultural level or persons of certain professions? This can be expressed in:

- artificial reduction of literacy;

— neutralization of speech;

— imitation of another person's written speech;

- simulated writing of women, young or old man;

— imitation of the style of a particular author;

- imitation of speech of the person who more than once served imprisonment;

— imitation of a certain dialect;

— imitation of the speech of a particular person under stress.

With intentional change in the degree of literacy there is the presence of spelling mistakes, while respecting the complex syntax rules, correct and complex architectonics of writing. In this case, the following discrepancy may be observed: simple words are written with errors, but complex words - correctly. Based on the literacy assessment, a conclusion can be made about the educational level of the author of the letter.

3. Native language is determined (or language environment), the professional environment and the estimated region of residence of the author of the document.

4. The performer and the author can be different persons, because the texts could be rewritten or performed under the dictation of another person. Therefore, it is necessary to consider whether the author and performer of the text is one person. In cases where manuscripts are not performed by the author, there is usually a discrepancy of a low degree of handwriting development to a high level of development of lexical and stylistic writing skills, and vice versa.

5. To determine the gender of the person in the Russian Federation a method iteratively tested developed by the research institute of the Ministry of internal affairs is used. The essence of this method is the selection of a specific complex of 21 features of handwriting. These features are written down with putting down coefficients of statistical probability of frequency of their occurrence in handwriting of men and women. Then the coefficients are multiplied, and if the resulting product is greater than one, it means that the manuscript is likely to have been performed by 
a man, and if less than one - by a woman. The degree of probability of conclusion is approximately $90 \%$.

Handwriting studies allow us to determine the executor of an anonymous document with a high degree of accuracy, however, as mentioned earlier, the author and performer of an anonymous document might not coincide. To identify the diagnostic and identification features of the author of the document, the letter must be subjected to linguistic research.

The main objectives of the linguistic study of anonymous documents are:

- determination of the level of proficiency in the language of the author of the document;

- determination of the text creation situation;

- interpretation and explanation of meanings and origins of words, phrases, stable phraseological expressions (idioms);

- interpretation of primary and secondary meaning of language units;

- interpretation of the provisions of the text in order to determine which variants of understanding of these provisions are possible in today's discourse.

The conclusion of the study, carried out with the use of generally accepted norms of the Russian language, knowledge of textual and stylometry, morphological, syntactic, linguostatistical analysis, can be worn as a likely and categorical character. The form of expert conclusion depends on the size and informativeness of the material submitted for examination. Categorical conclusion about the authorship of the text is possible, if the text contains at least 500 words.

In order to establish, through linguistic expertise, the extremist nature of the texts submitted, verbal signs of incitement to racial, national, religious or social hatred or discord must be identified. Such a result can be achieved by determining the illocutive effect aimed at the formation of the addressee (reader, listener), extremist materials, and negative stereotypes in relation to certain groups of the population (social, national, political, ideological, etc.). The extremist text may contain statements of a positive nature, for example, justifying or glorifying such negative phenomena as genocide, repression, deportation, discrimination against certain groups of the population. 
The text can display many mental traits of its author. The choice and usage of linguistic tools allow us to assess the degree of anxiety of the author to make it an affective or normal condition, etc. In some cases, they allow you to diagnose some typical personality traits (e.g., temperament, women and men, psychopathic). ${ }^{1}$

Meaning may be expressed in the text directly or indirectly (e.g., direct statement and rhetorical question, which is an indirect statement; or direct and indirect inducement in the form of a question). Meaning can be present in the text in explicit and implicit (hidden) forms.

It should also be borne in mind that the meaning of a language message always has synergy, it does not come down to the sum of the meanings of its language units. Semantic content can be expressed directly or indirectly, formed as direct specific calls, and semi-vague hints, references to folklore, historical events, cultural phenomena, etc.

To illustrate the theoretical positions analyzed, let us cite an example of a specific study carried out by the authors of the paper.

In 2016, the authors produced a specialized complex handwriting, linguistic and psychophysiological study on samples of handwriting to make a psychological portrait of the author and performer submitted to the study of an anonymous note, addressed to the head of a construction organization, Mr. Ermak. Fragments of this letter are shown in Fig. 1 and Fig. 2 (enlarged image).

During linguistic examination elements of the semantic core of the note were identified, as well as basic characteristics for the author and performer, textual features that could be related to the author's features (text architecture, absence of red lines, small fields, stylized style of writing signs, the presence of specific syntactic and grammatical errors, speech speed, etc.). The methodology used was based on methodological recommendations applied by experts from the Federal Security Service and the Ministry of Internal Affairs of Russia. ${ }^{2}$

1 A.N. Baranov, Linguistic Examination of Texts: Theoretical Grounds and Practice, Science, Moscow 2013 [In Russian].

2 Yu.M. Dildin, V.V. Martynov, eds., Typical Expert Methods for Investigating Physical Evidence, vol. 1, Inter-Krim Press, Moscow 2010 [In Russian]. 


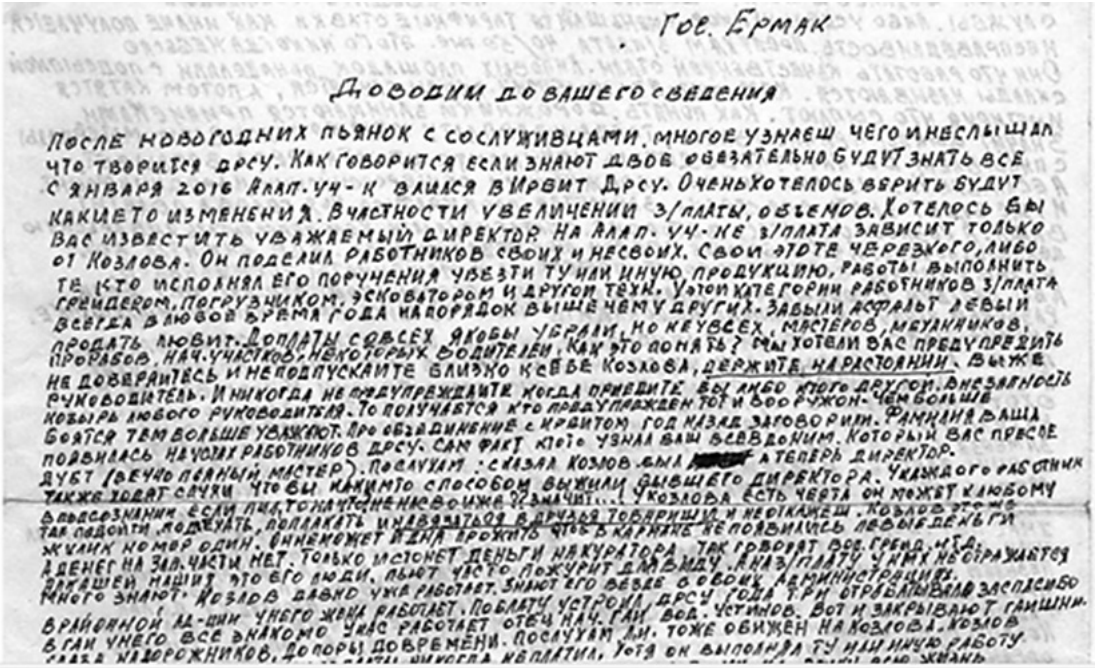

Figure 1. Anonymous letter

Source: A. Antropov.

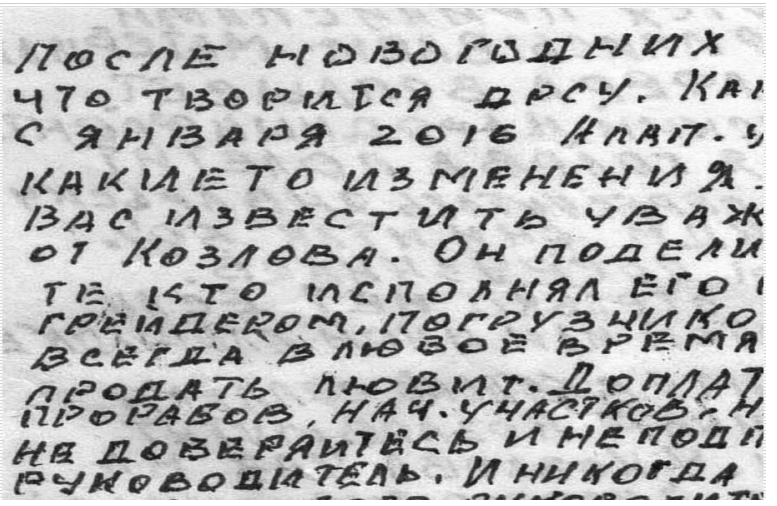

Figure 2. Enlarged image of the anonymous letter

Source: A. Antropov.

The presence of grammatical and punctuation errors is of an artificial nature, since visible brittle violations are allowed, while "difficult" words and phrases are written competently, without errors. The beginning of words with existing errors have signs of an unusual letter (knock- 
ing down factor - unreasonable stops of the writing device, slowness of the tempo of the letter).

The gender is male.

Nationality: Slav, Russian-speaking, school - Russian general education.

Education: above average - perhaps a technical school (probably technical or economic). High level of diligence, assiduity. Possession of the skills of stylized writing (imitation of the printed font), the degree of depletion of which is high (stability of graphology and insignificant degree of variation). It is highly likely that the stylized letter was formed during the years of training in a technical educational institution.

The writer's age is about 55-60 years.

Gesticulation is small, inactive.

Business ability: the writer thoroughly knows his work, but almost feels a personal insult, which can cause damage "on the sly". He believes that his services to the enterprise are not sufficiently assessed. He has worked for this company for more than 20 years (possibly one of the oldtimers). At some time in the past, there probably was an opportunity to become a middle manager, but, in fact, his influence declined or was lost. He was very well oriented in the hierarchy and system of the enterprise. The possible job of the writer - dispatcher, storekeeper, supply.

Functional duties are connected with material values - most likely it is a "materially responsible person". Through it passes information or the material values themselves. Perhaps he keeps personal diaries, collects a "dossier".

Personal traits: seclusion, perseverance, pedantry, systematic, accuracy, patience, while it is reliably established that this person is greedy, stingy and envious. He likes to spend time alone.

Probably a "bifurcation" in behavior. In public helpful and polite, in himself is secretive, suspicious, and very touchy. Polite, at work often communicates with persons above his office. Then cowardly and very cautious.

This type of personality can for years prepare revenge, calculate everything, collect information, facts, evidence. Such people, as a rule, strike so that the object does not know from whom the threat was coming. At the same time, such a person cannot be hypocritical and manipulative. He is unlikely to be able to attract to his "game" a large number of people. He will do everything himself and secretly.

Nowa Kodyfikacja Prawa Karnego 49, 2018

(C) for this edition by CNS 
Characteristics of nervous processes: cyclic - rapid internal excitability with loss of attention, then concentration in a state of calm. He is capable of concentrating in one direction in matters, thoughts, attachments. Writing the text of printed books speaks of schizophrenic inclinations in combination with complexes. Perhaps the author of the texts is undergoing treatment of a neurologist or psychiatrist.

Characteristics of the author of the anonymous message, obtained as a result of linguistic research, were presented to the management of the organization. As a result, the main suspect was identified, comparative samples of his handwriting (Fig. 3) were obtained, which were examined during the handwriting examination.

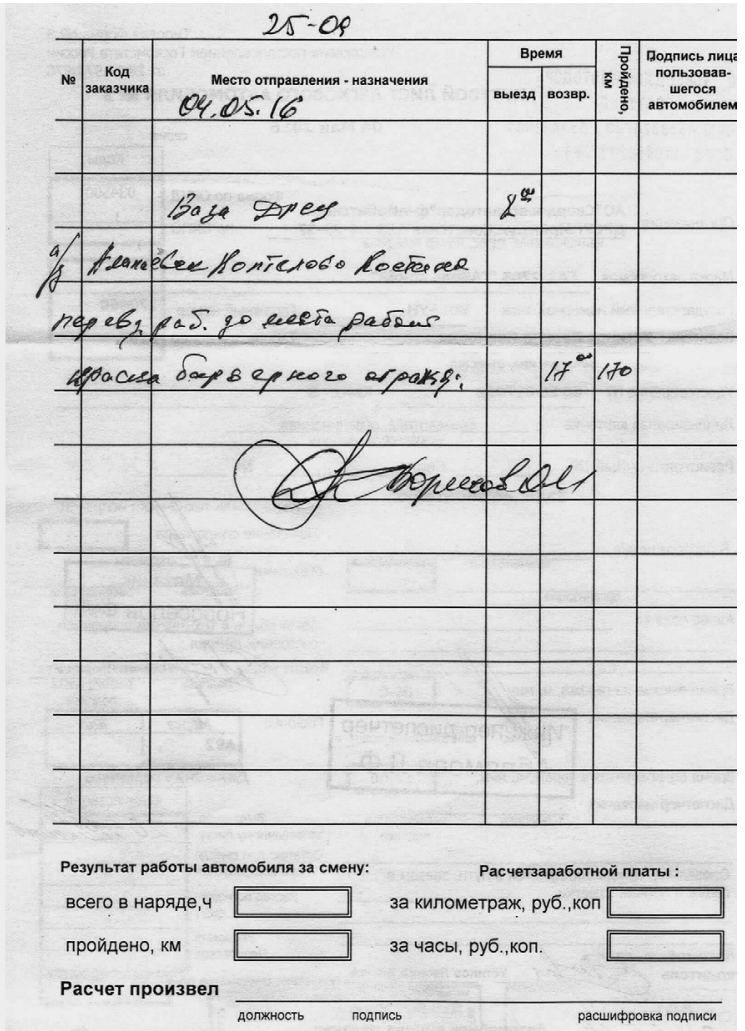

Figure 3. Handwriting samples on a waybill

Source: A. Antropov.

Nowa Kodyfikacja Prawa Karnego 49, 2018

(C) for this edition by CNS 
An expert was asked:

"Were the texts on the anonymous letter and the waybill performed by one person?"

As a result, a stable coincidence of the most pronounced cross-cutting particular features was established.

Letters "B" and "P" (Fig. 4).

1. Connection of movements when writing the elements of the letter " $\mathrm{B}$ " is interval.

2. The number of movements of performing the letter " $\mathrm{B}$ " is increased due to the presence of reflex strokes when the final part of the first element of the letter " $\mathrm{B}$ " is executed.

3. The shape of the movement when performing the upper part of the lowercase element of the letter "P" is angular.

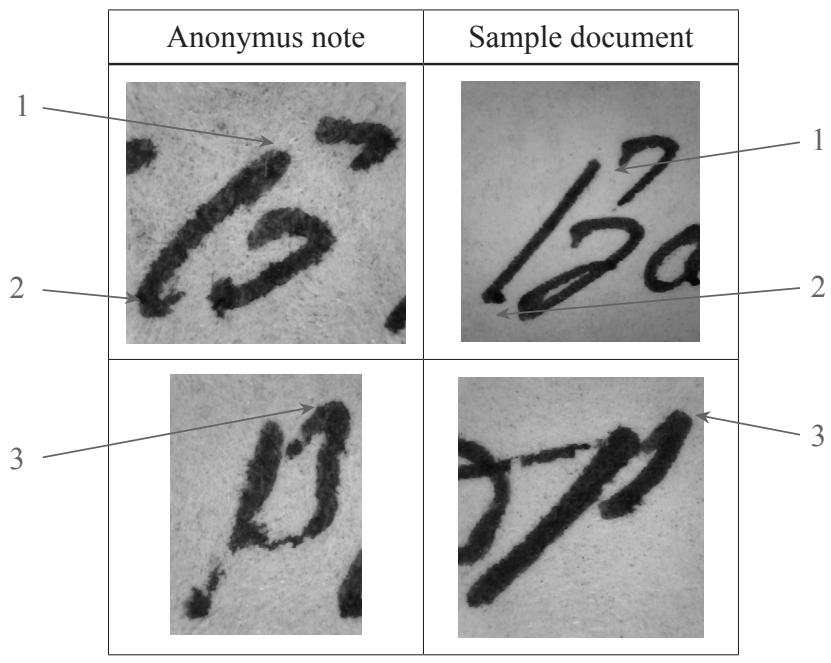

Figure 4. Comparison of letters "B" and "P"

Source: A. Antropov.

Letters "Y" and "Ж”" (Fig. 5).

4. The length of the movement when the first stroke of the letter "Y" is performed is reduced vertically.

5. The shape of the movement when the first and third elements of the letter "Ж” (it is a Cyrillic letter) are angular. 


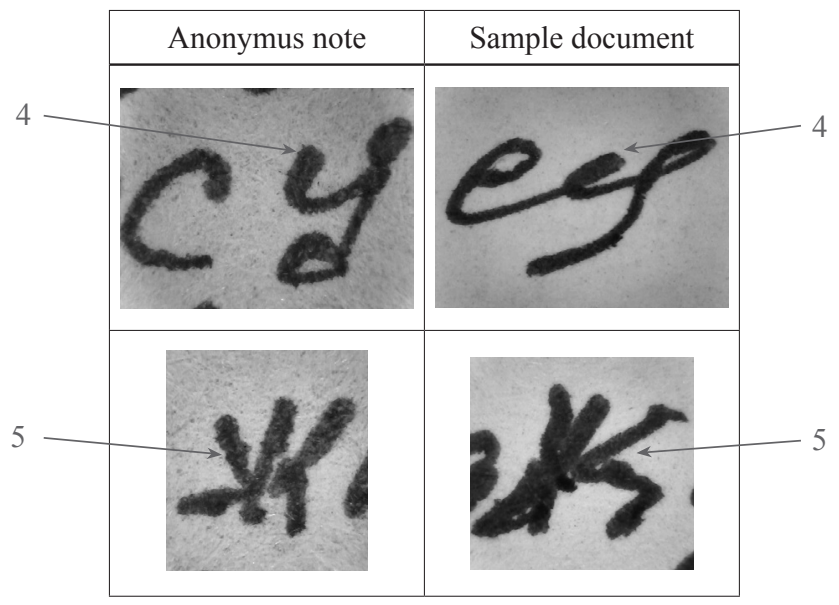

Figure 5. Comparison of letters "Y" and "Ж” Source: A. Antropov.

Letters "A", "O" and "T" (Fig. 6).

6. Connection of movements when writing the first and second elements of the letter " $\mathrm{A}$ " is interval.

7. The shape of the movements when the right side of the oval of the letter " $O$ " is executed is close to the straight.

8. Constructive complexity of movements when the letter " $\mathrm{T}$ " is performed is simplified in the form of a printed sign.

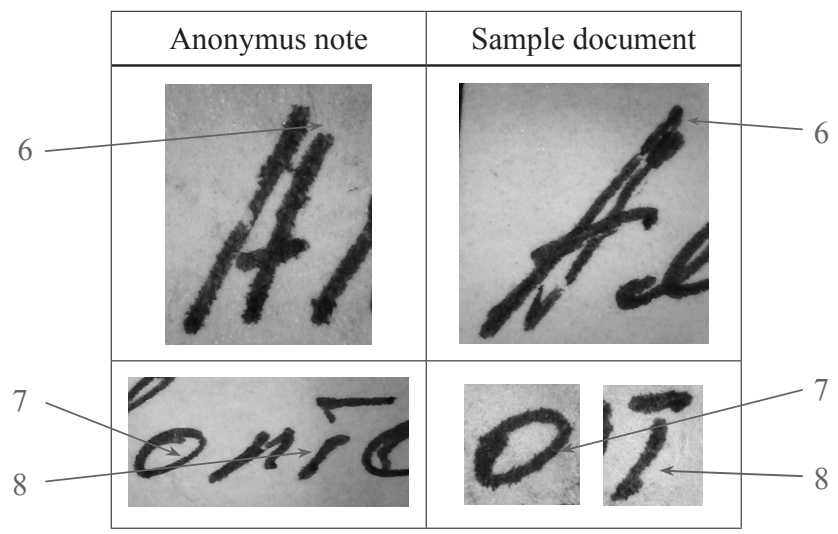

Figure 6. Comparison of letters "A" and "O"

Nowa Kodyfikacja Prawa Karnego 49, 2018 Antropov.

(C) for this edition by CNS 
On the basis of the conducted research the following conclusion was made: The texts of an anonymous letter and a waybill are executed by one person.

This indicates that the integrated application of research methods of documents makes it possible to accurately identify both the author and the performer of anonymous messages.

\section{References}

Baranov A.N., Linguistic Examination of Texts: Theoretical Grounds and Practice, Science, Moscow 2013 [In Russian].

Dildin Yu.M., Martynov V.V., eds., Typical Expert Methods for Investigating Physical Evidence, vol. 1, Inter-Krim Press, Moscow 2010 [In Russian].

\section{Summary}

The anonymous document as an object of forensic research has an information field that is subject to study by three groups of scientific disciplines: handwriting, technical research of documents and forensic linguistics. Stability and reproducibility characteristics of handwriting and written speech allows us to identify the author and performer of an anonymous message, as demonstrated by the example of the authors' practice.

Keywords: handwritten document, anonymous, handwriting examination, linguistic examination. 